\title{
Cascading Constrained 2-D Arrays using Periodic Merging Arrays
}

\section{Forchhammer, Søren; Laursen, Torben Vaarby}

Published in:

International Symposium on Information Theory 2003

Link to article, DOI:

10.1109/ISIT.2003.1228123

Publication date:

2003

Document Version

Publisher's PDF, also known as Version of record

Link back to DTU Orbit

Citation (APA):

Forchhammer, S., \& Laursen, T. V. (2003). Cascading Constrained 2-D Arrays using Periodic Merging Arrays. In International Symposium on Information Theory 2003 IEEE. https://doi.org/10.1109/ISIT.2003.1228123

\section{General rights}

Copyright and moral rights for the publications made accessible in the public portal are retained by the authors and/or other copyright owners and it is a condition of accessing publications that users recognise and abide by the legal requirements associated with these rights.

- Users may download and print one copy of any publication from the public portal for the purpose of private study or research.

- You may not further distribute the material or use it for any profit-making activity or commercial gain

- You may freely distribute the URL identifying the publication in the public portal

If you believe that this document breaches copyright please contact us providing details, and we will remove access to the work immediately and investigate your claim 


\title{
Cascading Constrained 2-D Arrays using Periodic Merging Arrays
}

\author{
Søren Forchhammer, Torben V. Laursen \\ Research Center COM, 371 \\ Technical University of Denmark \\ DK-2800 Lyngby \\ \{sf, tvl\}@com.dtu.dk
}

\begin{abstract}
We consider a method for designing 2-D constrained codes by cascading finite width arrays using predefined finite width periodic merging arrays. This provides a constructive lower bound on the capacity of the 2-D constrained code. Examples include symmetric RLL and density constrained codes. Numerical results for the capacities are presented.

The 2-D codes considered are shift invariant constraints of finite extent $(N, M)$. A constraint is defined by a set of admissible $N \times M$ configurations. The complement to this set is called the forbidden configurations. A configuration on an $n$ by $m$ rectangle is one having no forbidden configurations within the rectangle.
\end{abstract}

Let $F(n, m)$ be the number of admissible configurations on an $n$ by $m$ rectangle. Then the per symbol capacity, $C^{(2)}$ of the constraint is defined as follows.

$$
C^{(2)}=\lim _{n, m \rightarrow \infty} \frac{F(n, m)}{n m} .
$$

Calkin and Wilf [3] presented a method giving tight bounds on the capacity for first order constraints, but they do not apply when $N \geq 2$ and $M \geq 2$ [4]. Etzion [1] studied 2-D $\operatorname{SRLL}(d, k)$ constraints and gave methods for constructing a merging array $W$ given any two admissible arrays, $X$ and $Y$.

Instead of designing the fixed width merging array, $W$ after $X$ and $Y$, we construct $W$ first and then examine how $X$ and $Y$ may be constrained such that the (horizontal) cascading $W X W Y W$ is admissible. These merging arrays are simpler and easier to identify (e.g. for synchronisation).

Consider a 2-D array, $W$ of width $w \geq M-1$ and infinite height. This merging array is repeated at intervals of $m+w$ columns horizontally. This leaves arrays of width $m$ undefined in between two merging arrays. Let $X$ and $Y$ be 2-D arrays of width $m$ such that they can be cascaded to form an admissible configuration $W X W Y W$. Repeating this construction adding one array at a time the array may extend to define an admissible configuration in the entire plane.

Following Shannon [2] we describe the admissible arrays $W X W$ by a finite state source with states of height $n \geq N-1$.

The per symbol capacity for the resulting merged array is

$$
H=\frac{H_{W}(m)}{m+w}
$$

where $H_{W}(m)$ is the capacity of the $W$ boundary constrained array $W X W$ in which $X$ has a width of $m$.

Example $A-$ For (Symmetric RLL) SRLL $(d, k)$ we propose the following periodic merging array. Let $x$ be a row of $d 0 \mathrm{~s}$ followed by $d$ 1s. Let $x_{d}$ be $d$ identical rows of $x$ on top of each other. Then $W$ is given by alternating between $x_{d}$ and its bitwise negation. We note that the finite state source for
$W X W$ has $2 d$ phases since $W$ is periodic with period $2 d$. We can generalise the construction by offsetting the phase of say the right merging array. Let $W_{\phi}$ denote $W$ offset by the number of rows, $\phi$, maximizing the capacity, $H_{\phi}$, of $W X W_{\phi}$.

In Table 1 results are given for the $\operatorname{SRLL}(2,3)$ constraint. The periodic merging arrays have $w=4$ whereas Etzion's merging arrays have $w=12$. Since the $W$ boundary constrained arrays have 2 deterministic columns (beside $W$ ), we have compared bounds obtained using $m$ and $m+2$ columns, respectively.

\begin{tabular}{|crrrrr|}
\hline$m$ & 11 & 12 & 13 & 14 & 15 \\
\hline$\frac{H(m)}{m+12}$ & 0.126 & 0.131 & 0.135 & 0.139 & 0.143 \\
$\frac{H_{W}(m+2)}{m+6}$ & 0.0923 & 0.116 & 0.136 & 0.137 & 0.124 \\
$\frac{H_{\phi}(m+2)}{m+6}$ & 0.131 & 0.123 & 0.136 & 0.137 & 0.138 \\
\hline
\end{tabular}

Table 1: Lower bounds on capacity of $\operatorname{SRLL}(2,3)$

Example $B-$ Given $N$ and $M$, the binary $(d, k)$ density constraint is defined by $d \leq \sum_{i=1}^{N} \sum_{j=1}^{M} x_{i j} \leq k$ where $0 \leq$ $d \leq k \leq N M$ and $x_{i j} \in\{0,1\}$.

Table 2 gives the capacity using a checkerboard periodic merging array with $w=2$ for the $(4,5)$ density constraint with $N=M=3$. The minimum width of a merging array for arbitrary arrays $X$ and $Y$ is not known. However by using a simple example we have shown that it can not be less than 8 . Hence we also give the 'lower bounds' (in parentheses) using this value in Table 2 . It is seen that the periodic merging array approach is superior in this example.

\begin{tabular}{|cccccc|}
\hline$m$ & 7 & 8 & 9 & 10 & 11 \\
\hline$\frac{H(m)}{m+8}$ & $(0.279)$ & $(0.291)$ & $(0.302)$ & $(0.312)$ & $(0.320)$ \\
$\frac{H_{W}(m)}{m \pm 2}$ & 0.353 & 0.360 & 0.372 & 0.382 & 0.389 \\
\hline
\end{tabular}

Table 2: Lower bounds on capacity for density $(4,5)$

\section{REFERENCES}

[1] T. Etzion, "Cascading methods for runlength-limited arrays," IEEE Trans. Inform. Theory, vol. 43, no. 1, pp. 319-324, 1997;

[2] C. E. Shannon, "A mathematical theory of communication," Bell Syst. Tech. J., vol. 27, pt. I, pp. 379-423, 1948; pt. II, pp. 623-656, 1948.

[3] N. J. Calkin and H. S. Wilf, "The number of independent sets in a grid graph," SIAM Journal of Discrete Mathematics, vol. 11, no. 1, pp. 54-60, Feb. 1998.

[4] S. Forchhammer and J. Justesen, "Entropy bounds for constrained two-dimensional random fields," IEEE Trans. Inform. Theory, vol. 45, no. 1, pp. 118-127, Jan. 1999. 